\title{
Rising ethnic diversity in the United States accompanies shifts toward an
} individualistic culture

\author{
Alex C. Huynh ${ }^{1,2}$, Igor Grossmann ${ }^{2}$ \\ ${ }^{1}$ Psychology Department, California State University, San Marcos, 333 South Twin Oaks Valley \\ Road, San Marcos, CA, 92096-0001, USA \\ ${ }^{2}$ Department of Psychology, University of Waterloo, Waterloo, Ontario, Canada N2L 3G1.
}

in press

Social Psychological and Personality Science

Last Updated: September 26, 2020

**This is a preprint of a manuscript currently in press at Social Psychological and Personality Science. This paper may not exactly replicate the final document that will be published.

Corresponding author: Alex Huynh; email: ahuynh@csusm.edu

Acknowledgments: We thank Michael Wohl, Richard Eibach, Steven Mock, and Hilary Bergsieker for their comments on the research ideas and assistance with a previous draft of the manuscript.

Data and materials availability: Pre-registration information and data for all studies are available on the open science framework at the following link: osf.io/ytqz4. Data collected in Studies $1 \mathrm{a}$ and $1 \mathrm{~b}$ are subject to the availability of data in the Integrated Public Use Microdata Series (IPUMS-USA), which is publicly available at https://usa.ipums.org/usa/

Author contributions: A.C.H. collected the data, performed the data analyses, and wrote the paper. I.G. performed analyses for Study $1 \mathrm{a}$ and $1 \mathrm{~b}$, and provided guidance and supervision over the research. All authors were involved in the development of the research ideas, hypotheses, and revisions of the manuscript. 


\begin{abstract}
We investigate the relationship between ethnic diversity and the rise of individualism in the United States during the $20^{\text {th }}-21^{\text {st }}$ centuries. Tests of the historical rates of ethnic diversity alongside individualistic relational structures (e.g., adults living alone, single/multi-child families) from the years 1950-2018 reveal that societal and regional rates of ethnic diversity accompanied individualistic relational structures. These effects hold above and beyond time series trends in each variable. Further evidence from experimental studies $(N=707)$ suggest that the presence of, and contact with, ethnically diverse others contributes to greater individualistic values (e.g., the importance of uniqueness and personal achievement). Converging evidence across societal, regional, and individual-level analyses suggests a systematic link between ethnic diversity and individualism. We discuss the implications of these findings for socio-cultural livelihood in light of the rising rates of ethnic diversity across the globe.

Keywords: Ethnic Diversity, Cultural Change, Cultural Cognition, Individualism
\end{abstract}


Some recent estimates in the U.S. suggest that non-white minority groups currently comprise nearly $40 \%$ of the country's population, an increase from roughly $28 \%$ in 2010 , and 25\% in 2000 (“U.S. Census Bureau Data," 2020a). Despite emerging evidence that ethnic diversity is affecting many aspects of our social lives (e.g, Cox, Lobel, \& Mcleod, 1991; Van der Meer \& Tolsma, 2009; Rychlowska et al., 2015; Putnam, 2007), a systematic investigation into its impact on cultural values remains largely unexplored. Here, we investigate how ethnic diversity in the U.S. may be shaping socio-cultural systems, arguing that ethnic diversity is contributing to rising rates of individualism on societal, regional, and individual levels.

Individualism (Hofstede, 1980; Triandis, 1996) remains one of the most frequently discussed cultural dimensions in the psychological literature. Aligning with prior researchers (e.g., Brewer \& Chen, 2007), we use the term individualism here to refer to how people define themselves and their relationship with others, and how they structure their livelihood in the social system. While individualism includes a range of operationalizations, we focus on the elements of individualism that include agency, self-representation, and values (see Oyserman, Coon, \& Kemmelmeier, 2002 for review; also, Markus \& Kitayama, 2010). Namely, individualism here refers to behaviors and values that reflect the importance of the self being a separate and unique individual, a belief in individual autonomy and independence, and the importance of self-fulfillment and self-achievement.

Past decades of research on cultural change consistently demonstrate that individualism is rising (e.g., Inglehart \& Baker, 2000; Greenfield, 2013; Twenge, Campbell \& Gentile, 2013). The rise in individualism is demonstrated both in the U.S. when examining preferences for uniqueness (Grossmann \& Varnum, 2015), as well as globally when evaluating shifts toward individualistic societal practices (e.g., living alone, smaller household sizes), and ratings of the 
importance of happiness and autonomy (Santos, Varnum, \& Grossmann, 2017). Theorists chiefly explained these trends in terms of corresponding sociodemographic shifts in wealth and urbanization (Greenfield, 2013; Grossmann \& Varnum, 2015). However, wealth and shifting geographical regions are not the only ecological forces with the potential to stimulate the rise of individualism. Here we argue that another ecological trend - the rapidly rising rates of ethnic diversity (e.g., Putnam, 2007)— should also be investigated.

Several theoretical models suggest ethnic diversity may contribute toward rising individualism. For example, the common in-group identity model (Dovidio, Gaertner \& Kawakami, 2003) suggests that interethnic contact encourages people to re-categorize and decategorize their self-identities. In terms of self-representation, de-categorization involves seeing oneself as separate from others, while re-categorization involves incorporating outgroup members into ingroups (Gaertner et al., 2016). These responses to interethnic contact involve weakened ingroup boundaries and views of the self as separate from others - both of which are facets of individualism (Oyserman et al., 2002). Additionally, the integrated threat theory (Stephan \& Stephan, 2000) suggest that ethnic diversity highlights social stratification and hierarchy. According to this theory, ethnic diversity signals competition over resources, which may underscore the importance of personal achievement, another facet of individualism.

The positive relationship between ethnic diversity and individualism can be further bolstered from a social-ecological perspective (Oishi \& Graham, 2010). For example, sociologists argue that ethnic diversity is associated with community withdrawal and greater selfisolation (Putnam, 2007). Reduced communal engagement reflects weakened social ties, which may strengthen individualistic behavior through an increased reliance on individual autonomy and independence. Indeed, theorizing around the psychological impact of residential mobility 
(Oishi, 2010) suggests that tenuous social relationships (i.e., weakened social ties) formed by frequent relocation of livelihoods fosters an individualistic culture as it creates a system of selfsufficiency and autonomy.

Research Overview. Past research suggest that the presence of ethnic diversity can promote multiple facets of individualism—e.g., individuated self-representations, selfachievement motives, and self-reliance and autonomy. Building on these theories, we hypothesized that the presence of ethnic diversity would promote individualism. In Studies 1a and $1 \mathrm{~b}$, we model the socio-ecological association between ethnic diversity and individualism, using historical U.S. databases to derive country-level rates (Study 1a) and regional rates (Study 1b) of ethnic diversity and individualistic relational structures. In Studies 2 and 3, we examine individual-level processes, measuring participant's endorsement of individualistic values following the experimental manipulations of ethnic diversity in their social network (Study 2), and imagined social interactions (Study 3).

\section{Studies $1 \mathrm{a}$ and $1 \mathrm{~b}$}

\section{Method}

Procedure and Materials. All data for Study 1 were obtained through the Integrated Public Use Microdata Series USA (IPUMS). The IPUMS integrates U.S. census data collected on a decennial basis (i.e., every 10 years) from 1850-2000, and the American Community Surveys every year from 2000 onward (Ruggles et al., 2020). In Study 1a, we examine the magnitude of the relationship between ethnic diversity and individualism across this time period through time-series methods, including non-parametric correlations as well as Autoregressive Integrated Moving Averages (ARIMA) models.

In Study 1b, we assess how ethnic diversity relates to individualism across U.S. states 
from the years 2000-2018. This approach allowed us to capitalize on the availability of data $(n=$ 969), and control for variability in ethnic diversity within each U.S. state.

Indices of Individualism. Prior cultural researchers argue that interpersonal structure in society - e.g., the strength of family ties, reflect the society's level of individualism (Markus \& Kitayama, 1991; Hamamura, 2012). Although not a comprehensive way to assess individualism, the way people structure their interpersonal livelihood can be one way of assessing country-level trends in individualism. Thus, borrowing previously used procedures (e.g., Grossmann \& Varnum, 2015; Bianchi, 2016), the following variables were chosen as country-level correlates of individualism: i. Proportion of adults (18 years and older) living alone; ii. Average household family size (reverse-coded); iii. Proportion of single-to-multi child families, iv. Divorce-tomarriage ratios. All computations were done with variables weighted at the person-level in the IPUMS dataset. Notably, variables such as divorce rates and smaller house hold sizes are positively correlated with other country-level indicators of individualism (Ogihara, 2017) and the four indices together showed good reliability $(r=.86 ; \alpha=.96)$. As a measure of overall individualism, we converted each measure to z-scores and averaged them together for each year.

Index of Ethnic Diversity. To compute a measure of ethnic diversity, we employed the ethnic fractionalization (EF) index (Alesina, Devleeschauwer, Easterly, \& Kurlat, 2003; Montalvo \& Reynal-querol, 2005). The measure of ethnic fractionalization utilizes the following formula to operationalize ethnic diversity:

$$
E F=\sum_{i=1}^{N} \pi_{\mathrm{i}}\left(1-\pi_{\mathrm{i}}\right)
$$

Where $\pi_{\mathrm{i}}$ represents the proportion of people who belong to an ethnic group $i$, and $N$ is the number of groups. The final computed score is interpreted as the probability that two randomly 
selected individuals within a sample population will not belong to the same ethnic group.

This measure of ethnic diversity is used in the economic, psychological, and sociological literatures to understand numerous social science phenomena (e.g., cultural change in emotional expressivity, Rychlowska et al., 2015; changes in labour markets, Barr \& Oduro, 2002). The measure reflects the degree to which a population is evenly distributed into different ethnic groups, with higher scores reflecting greater ethnic diversity. Drawing from the available categories in the U.S. census, we obtained the following overall ethnic group proportions: $\mathrm{i}$. White; ii. Black/African American; iii. American Indian; iv. Chinese; v. Japanese, vi. Other Asian or Pacific Islander; vii. Other race; viii. Two major races; ix. Three or more major races. The latter two categories were only available beginning from the 2000 census data. With this information, we applied the formula for computing EF to obtain a measure of ethnic diversity from 1950-2018 (Study 1a: $M=.387 ; S D=.083$. Skew: -1.63, Kurtosis $=1.30 ;$ Study $1 \mathrm{~b}: M=$ $.325 ;$ SD $=.155$. Skew: .404, Kurtosis $=-.047)$.

Socioeconomic status. Past research argues that socioeconomic status (SES) is a robust predictor of individualism (e.g., Santos et al., 2007). Therefore, in secondary analyses we simultaneously evaluated the role of socio-economic status and ethnic diversity for individualism. We obtained the median household income from the U.S. Census data as a measure of SES ("U.S. Census Bureau Data," 2020b) - see the supplemental online materials (SOM) for more details. The collected census data was available for the years 1990-2018. Prior to analysis, we standardized SES by converting the measure to z-scores.

Analytical approach. We analyzed the relationship between EF and individualism while both controlling and not controlling for time. Because ethnic diversity and individualism both increased over time, examining raw correlations observes whether there is cross-temporal 
dependence (e.g., Twenge et al., 2013). Nevertheless, correlations between two variables over time may yield spurious relationships because each variable may be correlated with a third “time" variable. To account for the latter possibility, we employed two routine detrending procedures whereby the linear relationship of each variable with itself is partialled out (Boxsteffensmeier, Freeman, Hitt, \& Pevehouse, 2014): first-order differencing and best-fit line linear detrending (see SOM for exact procedures). First-order differencing involving computing a difference score between each time point and the previous time point, thereby accounting for the autocorrelation of each variable. The best-fit line detrending approach involves computing a linear trend for each variable, and then accounting for the linear trend by working with its residuals. After detrending, the four indices of individualism continued to hang together well (differencing, $\alpha=.79$; best-fit line, $\alpha=.88$ ).

After examining the correlations, our second analytic approach was to use ARIMA models (Dhamo \& Puka, 2010), which allowed us to decompose the time series data into the auto-regressive, differencing (trend), and moving average components. The auto-regressive $(A R(p))$ component refers to the use of past values in the regression equation for the series $\mathrm{Y}$. In a time series variable, it accounts for past values in the data when assessing the current value. The auto-regressive parameter $p$ specifies the number of lags used in the model. The differencing component $(I)$ refers to the number of differencing. Finally, the moving average $(M A(q))$ component represents the error of the model as a combination of previous error terms $e_{t}$. The order $q$ determines the number of terms to include in the model.

We used an automated ARIMA algorithm (auto.ARIMA) within the forecast function in $R$. This automated algorithm searches through combinations of parameters and picks the set that optimizes model fit, comparing Akaike information (AIC) or Bayesian information criteria 
(BIC). In short, if the final model selected by auto.ARIMA includes our putative exogenous variable (in this case, ethnic diversity) then this suggests that this variable helps the model to achieve optimal fit to the data. As a final step, we cross-validated our results by using the data from the first five decades to build a forecasting model and used the information from the last 10 years to compare predicted scores of individualism to the existing data ${ }^{1}$.

In Study 1b, we followed Study 1a by integrating an ARIMA structure into a linear mixed model framework. Following related procedures for time-series analyses (Jebb \& Tay, 2017), we identified the autoregressive structure for the relevant time period (2000-2018) across all states and then performed a linear mixed model analyses with the determined covariance, with scores nested within states.

\section{Study 1a Results}

Preliminary inspection of data showed that EF and individualism did not follow a normal distribution and showed signs of heteroscedasticity, Shapiro-Wilks $>.718, p<.001$.

Consequently, we relied on non-parametric Kendall's tau $(\tau)$ correlation coefficients (Croux \& Dehon, 2010; Newson, 2002). The magnitude of the relationship between EF and the overall averaged measure of individualism was large, $\tau=.85$, as was the relationship between SES and individualism, $\tau=.83$. As Table 1 shows, when partialling out shared variance with time, the relationship between EF and individualism continued to show a consistent positive relationship. Ethnic diversity as a predictor was larger in magnitude than household income (see Table S1 in the SOM). Notably, the difference between non-detrended correlation and those obtained after

\footnotetext{
${ }^{1}$ We further manually inspected the fit via sarima package in $R$, to ensure our auto.arima results provided the most parsimonious model for the data. Additional details around detrending and ARIMA procedures are available in the SOM. Syntax and analytic output for Studies 1a and $1 \mathrm{~b}$ are available at https://osf.io/ytqz4
} 
best-fit line detrending was negligible ( $r$ diff $=.07$ ), whereas the difference to first-order differencing estimates was large $(r$ diff $=.62$ ), suggesting a large degree of shared variance in the auto-regressive components (i.e., immediate preceding values) rather than overall trend over time. To simultaneously account for these components, we turned to ARIMA models.

Table 1

Correlation coefficients for ethnic diversity with correlates of individualism

\begin{tabular}{lccc}
\hline $\begin{array}{l}\text { Correlates of } \\
\text { Individualism }\end{array}$ & Non-detrended & $\begin{array}{c}\text { First-order } \\
\text { differencing }\end{array}$ & $\begin{array}{c}\text { Best-fit line } \\
\text { detrending }\end{array}$ \\
\hline Divorce/marriage ratio & $.78^{* * *}$ & $.30^{*}$ & $.88^{* * *}$ \\
Adults living alone & $.67^{* * *}$ & .10 & $.46^{* * *}$ \\
Smaller family size (rev) & $.83^{* * *}$ & .25 & $.81^{* * *}$ \\
Single-multi child family & $.78^{* * *}$ & .16 & $.72^{* * *}$ \\
\multicolumn{1}{c}{ Averaged measure: } & $.85^{* * *}$ & .23 & $.78^{* * *}$ \\
\hline
\end{tabular}

Note. Correlations represent the Kendall's $\tau$ coefficients. Family size was reverse coded so that all positive numbers indicate a positive association with the correlates of individualism. ${ }^{\dagger} p<.10 ;{ }^{*} p<.05 ;{ }^{* *} p<.01 ;{ }^{* * *} p<.001$

Consistent with the regression results, auto.ARIMA procedure indicated that a model with one auto-regressive component (i.e., the immediate preceding value)—ARIMA $(1,0,0)$ and a significant positive contribution of the ethnic diversity index, $B=9.86, S E=1.85, t=5.33$, $p<.001$, provides the best fit to the data:

$$
y_{t}(\text { individualist societal structure })=-3.85+0.92 y_{t-1}+9.86 x+e_{t}
$$

This model estimation suggests that ethnic diversity contributed to explaining the average individualist structure in the U.S. society above and beyond the temporal autocorrelations observed for average individualist structure.

We also ran an alternative set of analyses where we set ethnic diversity as the dependent variable and individualist structure as an exogenous predictor. The results of this automated forecasting procedure indicated a poorer model fit $(\mathrm{AIC}=115.68, \mathrm{BIC}=104.5)$, compared to the fit of the model above $(\mathrm{AIC}=7.95, \mathrm{BIC}=0.99)$, also showing that individualist structure was 
cross-temporally related to more ethnic diversity, $B=0.08, S E=0.01, t=13.75, p<.001$. The model fit results suggest that a model with ethnic diversity as a predictor rather than dependent variable is more likely to optimally reflect the structure of the data. Running an ARIMA model with both ethnic diversity and household income as simultaneous predictors from 1990-2018 dataset indicated a marginal effect of ethnic diversity, $B=3.50, S E=1.65, t=2.12, p=.051$, but no significant effect of income, $B=0.00002, S E=0.00001, t=1.68, p=.114$.

Forecasting results. As the left panel in Figure 1 shows, the estimated forecast (vs. observed data) for individualism was very close to obtained data from the last decade.
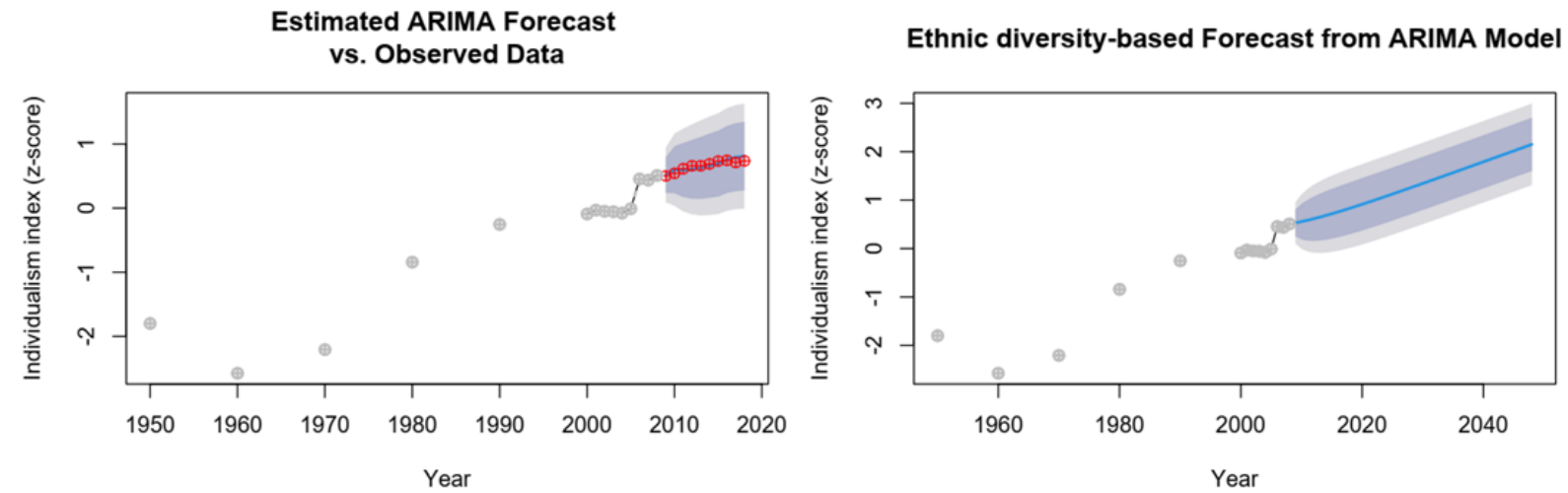

Figure 1. Ethnic diversity-based forecast for individualistic structure in a society from an ARIMA model. Confidence bands represent $80 \%$ and $95 \%$ intervals. Left panel: Cross-validation of the forecasted model against the observed individualist structure data from the last decade (red dots). Right panel: Forecasted direction of future trends in individualism with ethnic diversity as an exogenous predictor.

Additionally, we forecasted individualism for 3 decades after the last data point in our time series. Using the automated ARIMA algorithm, we also identified the best function for the ethnic diversity data, which we used to estimate the subsequent 30 data points. In turn, we used this estimated data in conjunction with the individualist structure data to forecast the further development of individualism. Results of this model suggest that individualism structure will continue to increase over the next several decades (see the right panel in Figure 1). These 
forecasts enable a test of this theoretical model against concrete future cultural trends.

\section{Study 1b Results}

Figure 2 depicts a heat map reflecting the change in EF over the years 2000-2018 for each U.S. State. Overall, the state with the highest mean-level of ethnic diversity was Hawaii ( $M$ $=.79, S D=.005)$, and the lowest was Maine $(M=.07, S D=.02)$. To assess the association between ethnic diversity and measures of individualism across all states, we first identified the optimal time series structure for the dependent variable. Automated ARIMA analyses suggested one autoregressive component $(1,0,0)$. Therefore, in subsequent linear mixed analyses we specified this autoregressive covariance structure, treating responses nested within each U.S. state at the individual-level, with years (2000-2018) as a repeated measures variable. Overall, the association between ethnic diversity and individualism was significant, $B=1.29, S E=0.44$, $t(917)=2.91, p=.003$. Table S2 in the SOM presents the fixed effects results for each index of individualism.

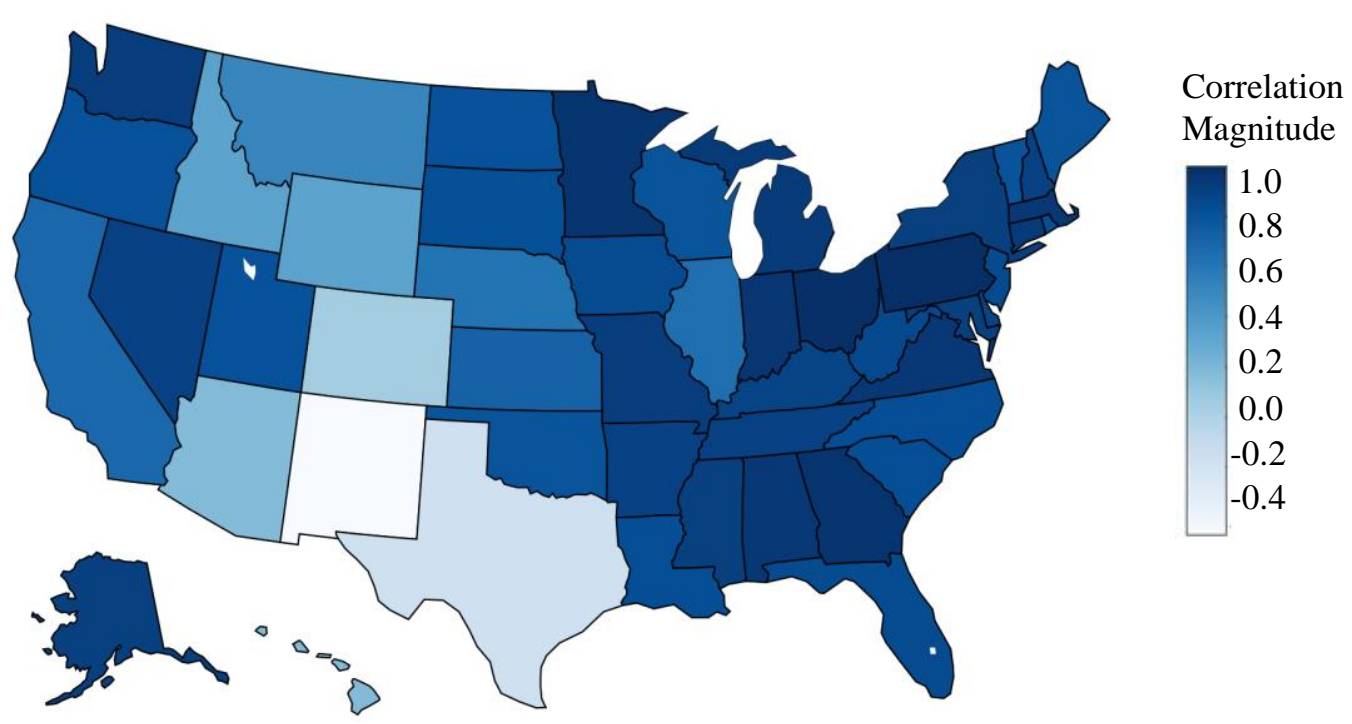

Figure 2. A heat map reflecting the change in EF over the years 2000-2018 for each U.S. state. Colors correspond to the Kendall Tau coefficients between ethnic fractionalization and years for each state, showing that while states have become more diverse over the past two decades, this was not a uniform shift for all states. 


\section{Study 2}

Study 1 results demonstrate that at the societal-level, ethnic diversity in a population is positively associated with correlates of individualistic relational structures. Notably, group-level analyses such as these may not always correspond to individual-level experiences ( $\mathrm{Na}$ et al., 2010). Thus, Study 2 transitions to investigating individual-level effects, using high-powered experimental designs that vary the perceptions and interactions with ethnic diversity in one's environment ${ }^{2}$. The study also included measures of community-level ethnic diversity and interethnic contact, which are reported in the SOM.

\section{Method}

Participants. As outlined in our pre-registration (https://osf.io/ytqz4) prior data yielded an average effect size of $f^{2}=.025$. A G* power analysis suggested a total sample size of approximately 325 participants to achieve a statistical power of .80 for 2 groups. We aimed to obtain 400 participants to account for exclusions. A total of 403 participants from Amazon's Mechanical Turk were recruited and compensated \$1 USD for their participation. Participants were excluded if their IP address was not in the U.S., and when they reported being more distracted than attentive to the study. The final sample size was 355 participants. Demographic information is presented in Table 2 .

Table 2

Demographics for Studies 2-3.

\begin{tabular}{lcc}
\hline & Study 2 & Study 3 \\
\hline Final N & 355 & 352 \\
Median Age & 35 & 34 \\
Median Income & $\$ 51-\$ 61 \mathrm{k}$ & $\$ 51-\$ 61 \mathrm{k}$ \\
Mean Social Class & 2.72 & 2.66 \\
Mean Political Ideology & 4.44 & 4.40
\end{tabular}

\footnotetext{
${ }^{2}$ A pilot study (reported in the SOM) motivated Studies 2-3 by demonstrating that greater ethnic diversity in a University classroom predicted greater individualistic self-representations.
} 


$\begin{array}{lcc}\text { Gender (f/m/non-binary) } & 199 / 152 / 4 & 222 / 128 / 2 \\ \text { Ethnicity } \% & 74.1 & 81.8 \\ \text { White } & 9.0 & 11.1 \\ \text { Black } & 8.2 & 6.0 \\ \text { Latino/Hispanic } & 7.0 & 2.0 \\ \text { East Asian } & 3.7 & 2.3 \\ \text { South Asian } & 0.0 & 1.1 \\ \text { Pacific Islander } & 0.8 & 3.4 \\ \text { Native American } & 1.7 & 1.1 \\ \text { Other } & \end{array}$

Note. Social class was measured on a scale from 1 (working class) to 5 (upper class). For income, participants were allowed to select from 9 categories, ranging from Less than $\$ 11,000$, to more than $\$ 80,999$. Political ideology was assessed on a scale ranging from 1 (very conservative) -7 (very liberal).

Procedure. Participants began the study by reporting their demographic characteristics, followed by our experimental manipulation. Adapting a measure from previous research (Schwarz et al., 1991), participants were randomly assigned to recall up to 3 ethnically different others in the social network (low-recall; $n=178$ ) or up to 12 ethnically different others (highrecall; $n=177)$. Participants were instructed to "Think about your own personal social relationship - that is, the specific people in your community with whom you have direct personal connections, such as your friends, acquaintances, neighbors, and coworkers." Participants were then prompted with the following:

Try to recall as many people among those social relationships whose ethnic background is different from your own. If you personally identify with multiple ethnic backgrounds, please think of people whose background is most different from yours. Please list up to [3 / 12] different people, providing the first name or initials of each person below.

The manipulation was designed to have participants in the high-recall condition perceive that their social networks were higher in ethnic diversity than those in the low-recall condition. Participants in the low-recall condition listed 2.94 others $(S D=.28)$, and those in the high-recall condition listed 8.41 others $(S D=3.87)$. To encourage reflection, a subsequent survey page asked participants to "think about the last interaction you had with one of the people you listed. 
Reflecting on this interaction, please write down a few sentences about who this person is (e.g., their relationship to you), what you talked about, and any topic(s) you may have discussed." To assess recall difficulty, participants were then asked to rate: "How difficult was it to recall others whose ethnic background is different than your own?" on a scale from 1 (not at all difficult) to 7 (extremely difficult). Participants then completed measures related to individualism.

Individualism measure. Individualism was assessed through an 17-item scale (Oyserman et al., 2002) ${ }^{3}$. The scale was designed to assess three facets of individualism: i. Personal uniqueness (e.g., "I enjoy being unique and different from others in many respects"); ii. Personal freedom and happiness (e.g., "Individual happiness and the freedom to attain it are central to who I am"), and iii. Personal achievement (e.g., "My personal achievements and accomplishments are very important to who I am"). Participants rated each item on a scale from 1 (strongly disagree) to 7 (strongly agree). Each facet showed good reliability: Personal uniqueness $(M=5.54 ; S D=.99 ; \alpha=.87) ;$ Personal freedom and happiness $(M=5.38 ; S D=.88$; $\alpha=.71)$; Personal achievement $(M=5.21 ; S D=1.02 ; \alpha=.81)$.

\section{Study 2 Results}

Participants in the high-recall condition reported significantly more difficulty recalling ethnically different others, $F(1,353)=7.68, p=.006, \eta^{2}=.02$. We decided to control for recall difficulty in subsequent analyses because our goal was to assess the relative effect of recalling more vs. less ethnic diversity in one's network, without the influence of difficulty. Results were similar without controlling for this variable and are reported on in the SOM. Notably, recall difficulty was negatively related to an overall averaged individualism measure, $r(355)=-.167, p$

\footnotetext{
${ }^{3}$ This scale included measures for collectivistic values, which were not predicted by our experimental manipulation - see SOM.
} 
$=.002$, suggesting that easily recalling ethnically diverse others was positively associated with a greater endorsement of individualistic values. As depicted in Figure 3, recalling up to 12 (vs. 3) ethnically different others resulted in a greater valuation of freedom and happiness, $F(1,352)=$ $6.08, p=.014, \eta^{2}=.017$, and a marginally greater endorsement of personal freedom and happiness, $F(1,352)=3.15, \mathrm{p}=.077 . \eta^{2}=.009$. We assessed the overall effect of condition on individualism, with individual scale items nested within participants. Results revealed that participants in the high-recall condition reported a greater endorsement of individualistic values $(M=5.52, S E=.024)$, relative to those in the low-recall condition, $(M=5.37, S E=.024), B=$ $.159, S E=.035, t=4.60, p<.001,95 \%$ CI $[.091, .227]$.

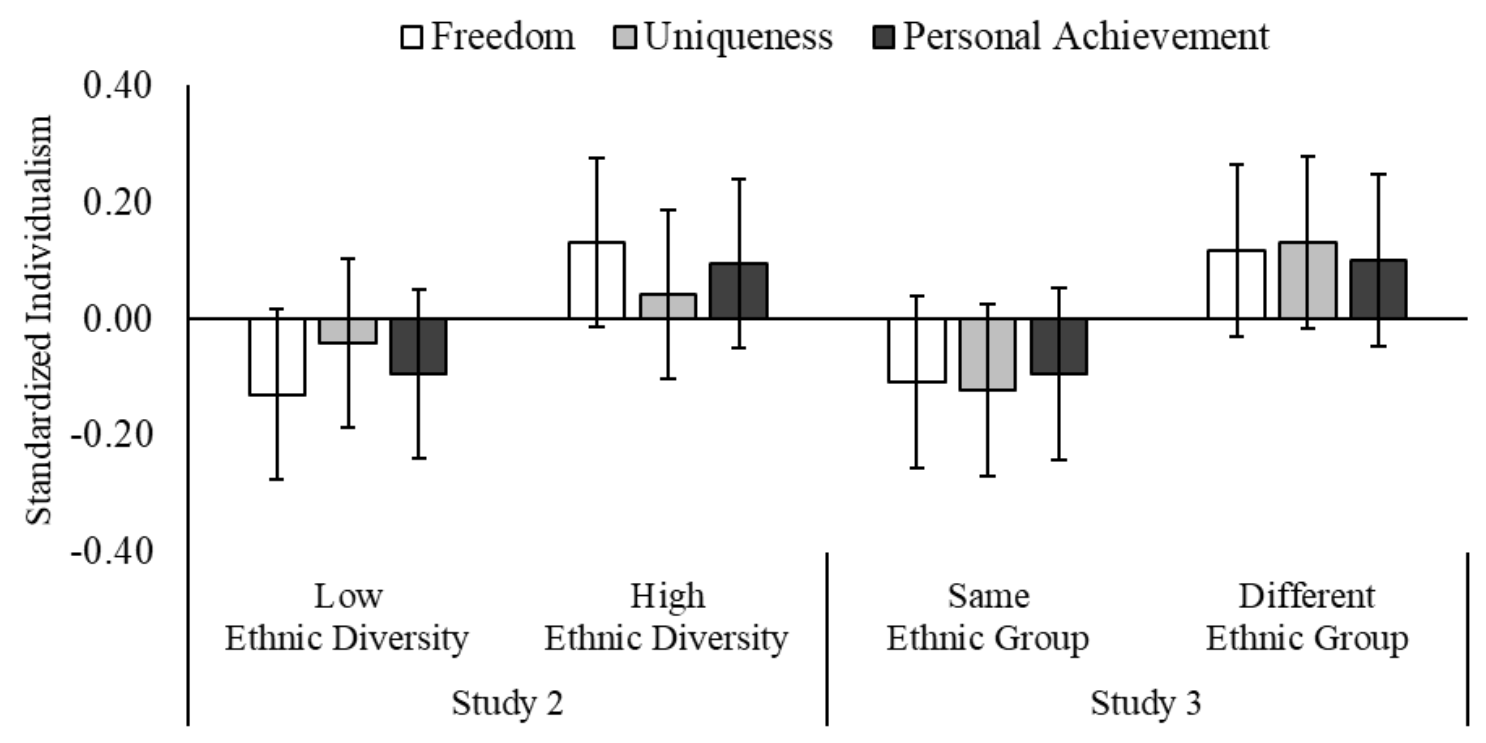

Figure 3. Variability in the endorsement of individualistic values by experimental manipulations of ethnic diversity in one's social network (Study 2) and social interactions (Study 3). Error bars reflect $95 \%$ confidence intervals.

\section{Study 3}

Study 2 provided experimental evidence that perceptions of higher ethnic diversity in one's social network increased individualistic cultural values — and in particular the valuation of 
individual freedom and happiness. Study 3 sought to replicate the observations in Study 2 by approaching exposure to ethnic diversity in an alternate way, assessing how people respond to interactions with ethnically diverse others. Including the same primary measures as those in Study 2 , Study 3 experimentally manipulated imagined interactions with those from the same vs. different ethnic groups.

\section{Method}

Participants. Planned sample size was determined using the same pre-registered rationale described in Study 2 (https://osf.io/ytqz4). We aimed to obtain 400 participants to account for exclusions and ensure adequate power. A total of 403 participants from Amazon's Mechanical Turk were recruited and compensated \$1 USD for their participation. Following the identical exclusion criteria used in Study 2, a final sample size consisted of 352 participants. Full demographic information is presented in Table 2 .

Procedure. Participants began the study by reporting their demographic characteristics. Participants were then randomly assigned to a prompt adapted from previous imagined interethnic contact research (Turner, Crisp, \& Lambert, 2007), in which they were asked to imagine interacting with a gender-matched other who was from the same $(n=182)$ or different $(n=170)$ ethnic background than their own. Those who identified as non-binary were presented with a random gender. Specifically, participants were told that they should "take a minute to imagine yourself meeting a person for the first time. The following is a description of the individual:"

This person lives in your local community, and is a [male/female] who is roughly the same age as you. This person is from [the same/ a different] ethnic background than your own.

Participants were asked to "take a moment to imagine this person. Think about their appearance, 
a conversation you might have, and the setting for where you might be." To encourage participants to immerse themselves in the imagined interaction, participants were also asked to "Describe in a few sentences the scene that you imagined yourself in. Where were you? What type of conversation did you have?" Upon completing their written response, participants completed measures of individualism.

Individualism measure. Individualism was assessed using the identical measure and scales in Study 2. Each facet of the scale showed good reliability: Personal uniqueness ( $M=$ 5.56; $S D=.96 ; \alpha=.84) ;$ Personal freedom/happiness $(M=5.39 ; S D=.92 ; \alpha=.73)$; Personal achievement $(M=5.22 ; S D=1.04 ; \alpha=.82)$.

\section{Study 3 Results}

As depicted in Figure 3, participants imagining interacting with an ethnically different (vs. same) person reported significantly greater valuation of uniqueness, $F(1,350)=5.74, p=$ $.017, \eta^{2}=.016$, freedom and happiness, $F(1,350)=4.53, p=.034, \eta^{2}=.013$, and marginally greater valuation of personal achievement, $F(1,350)=3.37, p=.067, \eta^{2}=.010$. Following Study 2 , we assessed the overall effect of condition on individualism using the same procedures. Results demonstrated that participants imaging interacting with an ethnically diverse person reported higher values on the individualism items $(M=5.59, S E=.025)$, relative to those imagining interacting with someone from the same ethnic background $(M=5.38, S E=.024), B=$ $.208, S E=.034, t=5.92, p<.001,95 \%$ CI $[.139, .277]$.

\section{Discussion}

In three studies, across different levels of measurement and analysis, we find converging evidence that ethnic diversity accompanies individualistic relational structures and increases the endorsement of individualistic values. Historical rates of ethnic diversity across different U.S. 
regions reveal this association (Study 1), as do individual level reports of interethnic contact (Studies 2-3). The results of the forecasting model in Study 1 suggests that the trend toward increasing individualism will likely continue over the next several decades, assuming continuing growth in ethnic diversity.

The effects observed in our studies are modest in size, but they identify ethnic diversity as a predictor of rising individualism, joining several other sociodemographic predictors of cultural change (e.g., urbanization, socioeconomic status; Greenfield, 2013; Grossmann \& Varnum, 2015). Given the breadth of information in cultural psychology on individualism (Li, 2002; Markus \& Kitayama, 1991; Triandis, 1996), the present research provides a means for researchers to anticipate how people will respond to the projected increase in rates of ethnic diversity. That is, future increases in ethnic diversity will likely be accompanied by an increasing societal emphasis on individualistic dimensions- e.g., individual autonomy and a greater preference for uniqueness (Oyserman et al., 2002). The present results also hold implications for mental health and well-being, as individualistic values impact what factors contribute to greater happiness (e.g., work-life balance, Haar et al., 2014; Markus \& Kitayama, 2010), and contribute to progressive connotations such as an increase in pursuing equality (e.g., Huppert et al., 2018).

\section{Limitations and Future Directions}

As with many psychological studies, the interpretations of results are limited by the methodology taken to assess the underlying constructs. The present studies operationalize ethnic diversity through ethnic fractionalization, although it could have been measured through other methods such as ethnolinguistic diversity—which considers language differences within groups (Alesina et al., 2003). There is evidence that different ways of operationalizing ethnic diversity captures different constructs and predict different outcomes (e.g., Montalvo \& Reynal-Querol, 
2010). Additionally, while the primary argument in this paper focuses on how ethnic diversity contributes to individualism, it is plausible that the relationship may be bidirectional (e.g., see research on residential mobility; Oishi, 2010). For example, ethnic diversity may increase individualism, which in turn may encourage traveling and exposure to greater ethnic diversity.

Given that moderns levels of ethnic diversity in the U.S. represent a specific culmination of a long history of colonization, institutional slavery, wars, and labor demands (Perez \& Hirschman, 2009), future research may benefit from exploring the observed association between ethnic diversity and individualism beyond the U.S. With recent evidence showing that ethnic diversity is increasing at exponential rates across the globe ("International Migration Report," 2017), and the simultaneous rise in individualism (Santos et al., 2017), it is possible that the associations between ethnic diversity and the rise of individualism would replicate in other countries, too. At the same time, it is possible that the relationship between ethnic diversity and individualism may be bounded by the endorsement of individualism as a superordinate cultural value in the society. Because salience of a national identity promotes the endorsement of dominant cultural values (Jettten, Postmes, \& McAuliffe, 2002), dominant cultural norms may shape the processes by which ethnic diversity affects cultural change. If ethnic diversity operates by promoting identification with one's group, it may result in a stronger endorsement of individualism in countries where that is the dominant cultural norm, but a different cultural value (e.g., collectivism) in countries with different norms. Addressing these competing possibilities via a cross-temporal study of ethnic diversity and individualism in different societies will be fruitful areas for future research.

In addition to cross-cultural replication, understanding the mechanism behind the association between ethnic diversity and individualism remains a key topic of study for future 
research. Beyond the possibility of ethnic diversity promoting dominant cultural values (Jetten et al., 2002), ethnic diversity may also highlight group-based identification, strengthening social ties for ingroup members. If this is the case, individualism would not be the only factor promoted by rise of ethnic diversity, with a potential for simultaneous increases in other cultural factors such as family-orientation or collectivism writ large. Additional analyses in Studies 2-3 in our SOM demonstrate possible effects on collectivism values, however these results were inconsistent. Effective tests of such mechanisms would require a combination of cross-cultural, cross-temporal, and psychometrically-rigorous experimental studies. 


\section{References}

Alesina, A., Devleeschauwer, A., Easterly, W., Kurlat, S., \& Wacziarg, R. (2003).

Fractionalization. Journal of Economic Growth, 8, 155-194.

Barr, A., \& Oduro, A. (2002). Ethnic fractionalization in an African labour market. Journal of Development Economics, 68, 355-379. https://doi.org/10.1016/S0304-3878(02)00017-2

Boone, A. (2005). Simulation of short-term wind speed forecast errors using a multi-variate ARMA $(1,1)$ time-series model. Power, 1-95.

Box-Steffensmeier, J. M., Freeman, J. R., Hitt, M. P., \& Pevehouse, J. C. (2014). Time series analysis for the social sciences. New York, NY: Cambridge University Press.

Bianchi, E. C. (2016). American individualism rises and falls with the economy: Cross-temporal evidence that individualism declines when the economy falters. Journal of personality and social psychology, 111, 567-584.

Cox, T. H., Lobel, S. A., \& Mcleod, P. L. (1991). Effects of ethnic group cultural differences on cooperative and competitive behavior on a group task. Academy of Management Journal, $34,827-847$.

Croux, C., \& Dehon, C. (2010). Influence functions of the Spearman and Kendall correlation measures. Statistical methods \& applications, 19, 497-515.

Dhamo, E., \& Puka, L. (2010). Using the R-package to forecast time series: ARIMA models and Application. In International Conference of Economic \& Social Challenges and Problems 2010 Facing Impact of Global Crisis.

Dovidio, J. F., Gaertner, S. L., \& Kawakami, K. (2003). Intergroup contact: The past, present, and the future, Group Processes \& Intergroup Relations, 6, 5-21.

Gaertner, S., Guerra, R., Rebelo, M., Dovidio, J., Hehman, E., \& Deegan, M. (2016). The 
common ingroup identity model and the development of a functional perspective: A cross-national collaboration. In The social developmental construction of violence and intergroup conflict (pp. 105-120). Springer, Cham.

Greenfield, P. M. (2013). The changing psychology of culture from 1800 through 2000. Psychological Science, 24, 1722-1731. https://doi.org/10.1177/0956797613479387

Grossmann, I., \& Varnum, M. E. W. (2015). Social structure, infectious diseases, disasters, secularism, and cultural change in America. Psychological Science, 26, 311-324. https://doi.org/10.1177/0956797614563765

Haar, J. M., Russo, M., Suñe, A., \& Ollier-Malaterre, A. (2014). Outcomes of work-life balance on job satisfaction, life satisfaction and mental health: A study across seven cultures. Journal of Vocational Behavior, 85, 361-373.

Hamamura, T. (2012). Are cultures becoming individualistic? A cross-temporal comparison of individualism-collectivism in the united states and Japan. Personality and Social Psychology Review, 16, 3-24. https://doi.org/10.1177/1088868311411587

Huppert, E., Cowell, J. M., Cheng, Y., Contreras-Ibáñez, C., Gomez Sicard, N., GonzalezGadea, M. L., . . Decety, J. (2019). The development of children's preferences for equality and equity across 13 individualistic and collectivist cultures. Developmental Science, 22, 1-15.

Inglehart, R., \& Baker, W. E. (2000). Modernization, cultural change, and the persistence of traditional values. American Sociological Review, 65, 19-51

International Migration Report. (2017). Retrieved from https://www.un.org/en/development/desa/population/migration/publications/migrationrep ort/docs/MigrationReport2017_Highlights.pdf 
Jebb, A. T., \& Tay, L. (2017). Introduction to time series analysis for organizational research: Methods for longitudinal analyses. Organizational Research Methods, 20, 61-94.

Jetten, J., Postmes, T., \& McAuliffe, B. J. (2002). 'We're all individuals': Group norms of individualism and collectivism, levels of identification and identity threat. European Journal of Social Psychology, 32, 189-207.

Li, H. Z. (2002). Culture, gender and self-close-other(s) connectedness in Canadian and Chinese samples. European Journal of Social Psychology, 32, 93-104. https://doi.org/10.1002/ejsp.63

Markus, H. R., \& Kitayama, S. (1991). Culture and the self: Implications for cognition, emotion, and motivation. Psychological Review, 98, 224-253.

Markus, H. R., \& Kitayama, S. (2010). Cultures and selves: A cycle of mutual constitution. Perspectives on Psychological Science, 5, 420-430. https://doi.org/10.1177/1745691610375557

Montalvo, J. G., \& Reynal-Querol, M. (2010). Ethnic polarization and the duration of civil wars. Economics of Governance, 11, 123-143. https://doi.org/10.1007/s10101-010-0077-8

Na, J., Grossmann, I., Varnum, M. E. W., Kitayama, S., Gonzalez, R., \& Nisbett, R. E. (2010). Cultural differences are not always reducible to individual differences. Proceedings of the National Academy of Sciences of the United States of America, 107, 6192-6197. https://doi.org/10.1073/pnas.1001911107

Newson, R. (2002). Parameters behind “nonparametric” statistics: Kendall's tau, Somers' D and median differences. The Stata Journal, 2, 45-64.

Ogihara, Y. (2017). Temporal changes in individualism and their ramification in Japan: rising individualism and conflicts with persisting collectivism. Frontiers in Psychology, 8, 695. 
doi: 10.3389/fpsyg.2017.00695

Oishi, S. (2010). The psychology of residential mobility: Implications for the self, social relationships, and Well-Being. Perspectives on Psychological Science, 5, 5-21. https://doi.org/10.1177/1745691609356781

Oishi, S., \& Graham, J. (2010). Social ecology: Lost and found in psychological science. Perspectives on Psychological Science, 5, 356-377.

Oyserman, D., Coon, H. M., \& Kemmelmeier, M. (2002). Rethinking individualism and collectivism: Evaluation of theoretical assumptions and meta-analyses. Psychological Bulletin, 181, 3-72.

Perez, A. D., \& Hirschman, C. (2009). The changing racial and ethnic composition of the US population: Emerging American identities. Population and development review, 35, 1-51.

Putnam, R. D. (2007). E pluribus unum: Diversity and community in the twenty-first century the 2006 johan skytte prize lecture. Scandinavian Political Studies, 30, 137-174. https://doi.org/10.1111/j.1467-9477.2007.00176.x

Ruggles, S., Alexander, T., Genadek, K., Goeken, R., Schroeder, M., \& Sobek, M. (2020). Integrated Public Use Microdata Series (IPUMS) (Version 10.0) [Machine-readable database]. Minneapolis, MN: University of Minnesota.

Rychlowska, M., Miyamoto, Y., Matsumoto, D., Hess, U., Gilboa-Schechtman, E., Kamble, S., ... \& Niedenthal, P. M. (2015). Heterogeneity of long-history migration explains cultural differences in reports of emotional expressivity and the functions of smiles. Proceedings of the National Academy of Sciences, 112, E2429-E2436.

Santos, H. C., Varnum, M. E. W., \& Grossmann, I. (2017). Global Increases in Individualism. Psychological Science, 28, 1228-1239. https://doi.org/10.1177/0956797617700622 
Schwarz, N., Bless, H., Strack, F., Klumpp, G., Rittenauer-Schatka, H., \& Simons, A. (1991). Ease of retrieval as information: Another look at the availability heuristic. Journal of Personality and Social Psychology, 61, 195-202. https://doi.org/10.1037//00223514.61.2.195

Stephan, W. G., \& Stephan, C. W. (2013). An integrated threat theory of prejudice. In S. Oskamp (Ed.), Reducing prejudice and discrimination (pp. 23-45). Mahwah, NJ: Erlbaum.

Triandis, H. C. (1996). The psychological measurement of cultural syndromes. American ethnic diveristy Psychologist, 51, 407-415. https://doi.org/10.1037/0003-066X.51.4.407 ethnic diversity

Turner, R. N., Crisp, R. J., \& Lambert, E. (2007). Imagining intergroup contact can improve intergroup attitudes. Group Processes and Intergroup Relations, 10, 427-441. https://doi.org/10.1177/1368430207081533

Twenge, J. M., Campbell, W. K., \& Gentile, B. (2013). Changes in pronoun use in American books and the rise of individualism, 1960-2008. Journal of cross-cultural psychology, 44, 406-415.

U.S. Census Bureau. (2020a). Quick Facts. Retrieved from https://www.census.gov/quickfacts/fact/table/US/PST045218

U.S. Census Bureau Data (2020b). Historical Household Income Tables. Retrieved from https://www.census.gov/data/tables/time-series/demo/income-poverty/historical-incomehouseholds.html

Van der Meer, T., \& Tolsma, J. (2014). Ethnic Diversity and Its Effects on Social Cohesion. Annual Review of Sociology, 40, 459-478. https://doi.org/10.1146/annurev-soc-071913043309 


\section{Supplementary Online Materials for}

Rising ethnic diversity in the United States accompanies shifts toward an individualistic culture

Alex C. Huynh ${ }^{1,2}$, Igor Grossmann ${ }^{2}$

This supplemental file includes the following additional information and figures/tables:

- Study 1a analytic procedures - Page 2

- Studies 2 and 3 additional measures of ethnic diversity and interethnic contact - Page 3

- Study 2 analyses without controlling for recall difficulty - Page 5

- Studies 2 and 3 analyses of collectivistic values - Page 6

- Socioeconomic status as a predictor of individualism - Page 7

- Supplemental pilot study (Methods/Results) - Page 8

○ Figure S1: Ethnic diversity rates (detrended and non-detrended) from 1950-2018

○ Figure S2: Cross-lagged panel analyses in Supplemental Pilot Study

○ Figure S3: Indirect mediation pathway for Studies 2 and 3

- Table S1: Correlation coefficients for ethnic diversity and correlates of individualism in Study 1a

○ Table S2: Fixed effect results for ethnic diversity and socioeconomic status in Studies $1 \mathrm{a}$ and $1 \mathrm{~b}$ 


\section{Study 1a analytic procedures}

As described in the main text, Study 1a involved analysis with detrended data, followed by an analysis with autoregressive integrated moving averages (ARIMA). Below, we provide some additional information about the detrending process and information around ARIMA analyses.

Detrending analyses. To assess the relationship between ethnic fractionalization and individualism, we sought to detrend relevant variables and assess their correlations. Detrended correlations show whether overall trends in variable A from time $t$ to $t+1$ correspond to similar changes in variable B over the same time segment. We elected two different methods to detrend the data: first-order detrending and best-fit line detrending. First-order detrending involves removing first-order autocorrelations by computing the difference score between each year. Thus, for each variable (ethnic diversity, household income, and individualistic relational structures - see Table 1 in the main text), we standardized the variable by computing $\mathrm{z}$-scores and then computed a difference score between each year. Resulting analyses for the first-order detrending method is an analysis of the change in time between years within each variable. The second method used was best-fit line detrending, which involved computing the least-squares fit of a linear trend in the data, and subtracting that resulting function from the data. This process was completed using the 'pracma' $\mathrm{R}$ package and was done for each variable of interest. Figure S1 depicts the resulting ethnic diversity data when detrended and not detrending.

Arima analyses. For interested readers looking to understand ARIMA analyses further, we reference Jebb \& Tay (2017) for a good discussion of decomposing time-series analyses into its components (including ARMA analyses and autocorrelation analyses). As described in the main paper, ARIMA analyses allow for the decomposition of data into different components: $(A R(p))$ the autoregressive component; the differencing component ( $I)$; and the moving 
average $(M A(q))$ component. We used an automated ARIMA algorithm (auto.ARIMA) within the forecast function in $R$ to identify the optimal forecasting function to our data when including relevant regressor (EF) as a predictor. This automated algorithm searches through combinations of order parameters and picks the set that optimizes model fit criteria, comparing Akaike information criteria (AIC) or Bayesian information criteria (BIC) of respective models.

Using the data provided, the auto.arima function attempts to fit the best model (lowest AIC) by testing combinations of possible models different components of the ARIMA (see: https://otexts.com/fpp2/arima-r.html for a description of the procedures used). In short, the values of $\mathrm{p}$ and $\mathrm{q}$ are then chosen by minimising the AIC after differencing the data $\mathrm{d}$ times. As described by the R package, "rather than considering every possible combination of $\mathrm{p}$ and $\mathrm{q}$, the algorithm uses a stepwise search to traverse the model space."

As reported in the main text, the final model selected by auto.ARIMA included ethnic diversity, suggesting that it helped the model to achieve optimal fit to the data. We crossvalidated the results via visual inspection of diagnostics (autocorrelation function plots and Lijun-Box tests) via sarima function in R (see https://osf.io/ytqz4 for the visual inspection analyses).

\section{Study 2 and 3 additional measures of ethnic diversity and interethnic contact}

In both studies 2-3, participants were asked to provide a zip code for where they currently lived. Zip code information was used through the U.S. census to pull the regional level ethnic group population distributions for each participant. As this data was provided from the U.S. census database, the ethnic groups were the same as those used in Study 1 in the main text. Using the distribution, a measurement of participant's regional ethnic diversity was computed using the index of ethnic fractionalization described in the main text. For Study 2: Mean $E F=.416, S D=$ 
.202. For Study 3: Mean $E F=.410, S D=.204$.

In both studies, participants also self-reported interethnic contact with 4-items. The four items were the following: i. In my immediate social network, many of the people I know are from another racial/ethnic background; ii. I frequently have contact with people from another racial/ethnic background; iii. Many of the people I socialize with are from a different racial/ethnic background than my own; and iv. I do not know many people that are from a different racial/ethnic background than my own (reverse-coded). Participants responded on a scale from 1 (strongly disagree) to 7 (strongly agree). In Study 2: $M=4.74, S D=1.59, \alpha=.90$. In Study 3: $M=4.65, S D=1.58, \alpha=.87$

Because the sample and measurements from Study 2 and 3 were similar, the studies were combined to assess the relationship between regional ethnic diversity, reports of interethnic contact, and the endorsement of individualistic values. Namely, we were interested in testing whether local levels of ethnic diversity were positively associated with participant's endorsement of individualistic values. Moreover, we were interested in whether this relationship was driven by an increase in interethnic contact. We performed a mediational analysis, testing whether the relationship between local levels of ethnic diversity and a participant's endorsement of individualism was statistically accounted for by the participant's reports of interethnic contact.

As depicted in Figure S3, regional ethnic diversity was not directly related to the overall endorsement of individualism, $r(706)=.039, p=.301$; however, reported interethnic contact was significantly related to the overall endorsement of individualism, $r(706)=.24, p<.001$, and was also significantly related to how ethnically diverse their local area is, $r(706)=.27 p<.001$. A test of the indirect mediation was significant $95 \% \mathrm{CI}[.17, .38]$, suggesting that ethnically diverse areas facilitate greater self-reported interethnic contact, which in turn contributes to a greater 
endorsement of individualistic values. Taken together, these results suggest that ethnic diversity in one's community can contribute to greater individualistic values, but only if people take the opportunity to engage in interethnic contact.

\section{Studies 2 and 3 analyses of collectivistic values}

As reported in the main text, Studies 2 and 3 incorporated a cultural measure that included collectivistic values (Oyserman et al., 2002). Mirroring the values of individualism, collectivism was measured with a 19-item scale that involved three facets of collectivism: i. Sense of common in-group fate (e.g., "It is important to me to think of myself as a member of my religious, national, or ethnic group"); ii. Familialism (e.g., "Family is more important to me than almost anything else"); and iii. Interrelatedness (e.g., "To know who I really am, you must see me with members of my group"). The reliability of each facet was good. In Study 3: Sense of common in-group fate $(M=3.98 ; S D=1.43 ; \alpha=.88)$; Familialism $(M=5.23 ; S D=1.25 ; \alpha=$ $.90)$; Interrelatedness $(M=4.39 ; S D=1.16 ; \alpha=.80)$. In Study 4: Sense of common in-group fate $(M=3.78 ; S D=1.57 ; \alpha=.90) ;$ Familialism $(M=5.32 ; S D=1.27 ; \alpha=.90) ;$ Interrelatedness $(M$ $=4.42 ; S D=1.14 ; \alpha=.78)$. Across Studies 2 and 3, we tested whether there was a significant relationship between any of these subcomponents, with the ethnic diversity measure in participant's communities, their self-reported intergroup contact, and whether the experimental conditions predicted their collectivistic values.

In Study 2, none of the collectivism subcomponents were significantly related to the levels of ethnic fractionalization in their local communities, $|r s|<.018, p s>.734$. The Familialism subcomponent was significantly positively related to reports of interethnic contact, $r$ $=.116, p=.029$, but the other two subcomponents were not related to intergroup contact, $|r s|<$ 
$.053, p s>.321$, The experimental condition of recalling 12 (vs. 3) ethnically diverse others did not significantly predict any of the collectivism subcomponents, $F \mathrm{~s}<1.30, p \mathrm{~s}>.254$.

In Study 3, ethnic fractionalization in the local communities was significantly negatively related to the Familialism subcomponent, $r=-.112, p=.036$, but not to either of the other two subcomponents, $|r s|<.088, p s>.099$. None of the collectivism subcomponents were significantly related reports of interethnic contact, $|r s|<.042, p s>.427$. The experimental condition of imagining an interaction with an ethnically different (vs. same) other did not predict any of the collectivism subcomponents, $F \mathrm{~s}<2.44, p \mathrm{~s}>.120$.

\section{Study 2 analyses without controlling for recall difficulty}

Here we report the same analyses in the main text for Study 2, but without controlling for recall difficulty. Results revealed that people asked to recall up to 12 ethnically different others in their social networks, reported significantly marginally greater valuation of freedom and happiness, $F(1,353)=3.71, p=.055, \eta^{2}=.010$. The relationship between condition and valuing personal freedom and happiness dropped away from trending toward significance $F(1,353)=$ $1.88, p=.172 . \eta^{2}=.005$. We assessed the overall effect of condition on individualism by pooling together all 17 individualism items and nesting the individual items within individuals. Results revealed were similar to that reported in the main text, showing that participants in the high ethnic diversity recall condition reported greater endorsement of individualistic values $(M=$ $5.50, S E=.024)$, relative to those in the low ethnic diversity recall condition, $(M=5.39, S E=$ $.024), B=.112, S E=.035, t=3.24, p<.001,95 \%$ CI $[.044, .179]$.

\section{Socioeconomic Status as a predictor of individualism}

Given that past research suggests socioeconomic status (SES) is a predictor of countrylevel individualism (e.g., Santos et al., 2017), we also assessed the relationship between 
socioeconomic status and individualism in all of our main studies, expecting to find a significant relationship while hypothesizing that the relationship between ethnic diversity and individualism effect would persist even when accounting for measures of socioeconomic status. As reported in the main text, Studies 1a and 1b operationalized SES as the median household income for each available year across the country in Study 1a and for each year for each U.S. State in Study $1 b$. Median household income was obtained through the U.S. census ("U.S. Census Bureau Data," 2020) and was only available from that particular census database for the years 1990-2018. Dollars were in current dollars (not adjusted for inflation). In Studies 2-3, SES was operationalized through a subjective measure of social class (Kraus, Piff, \& Keltner, 2011). Participants self-reported on a scale from 1 (lower class) to 5 (upper class), their agreement with the following statement: "With which social class group are you most identified, in terms of income and education?" Averaged measures of this variable are reported in Table 2 in the main text.

Using the methods described for Study 1a, the magnitude of the relationship between SES and individualism was large prior to detrending the data, Kendall's $\tau=.83, p<.001$. As shown in Table S1, when detrending the data, SES retained its positive relationship to individualism using one method, but not the other: first-order detrending: $\tau=.01$, best-fit line detrending, $\tau=.24$. In Study $1 \mathrm{~b}$, we simultaneously included the median household income for each U.S. state for each year in the model presented in the main text. That is, we included ethnic diversity and SES as simultaneous predictors of individualistic relational preferences across the country, while accounting for the variability of these factors in each U.S. State. Results revealed that overall, across the country, from the years 2000-2018, the association between ethnic diversity and individualism was significant, $B=.16, S E=.03, p<.001$, even when accounting 
for the relationship between SES and individualism, which was also significant, $B=.31, S E=$ $.03, p<.001$. A breakdown of the individualistic relational preferences is provided in Table S2.

In Study 2, SES did not significantly relate to any measure of the components of individualism $r$ 's $<.023$. Including self-reported socioeconomic status did not change the significant effects of any of these results. In Study 3, the measure of SES was significantly related to the measure of valuing personal achievement, $r(352)=.186, p<.001$, but not significantly related to either of the other two components, $r$ 's $<.074, p$ 's $>.166$. Including the measure of socioeconomic status did not change the significance of any of the relationship findings between ethnic diversity and any of the individualistic value subcomponents.

\section{Supplemental Pilot Study (Methods/Results)}

Between Study 1 and Studies 2-3 in the main text, we analyzed data from a pilot study described below. This study was not pre-registered, but was part of a University funded study with the first-author, to assess academic performance and well-being. Given that some variables in the dataset were related to our theoretical questions about ethnic diversity and individualism, we analyzed the dataset to assess their relationship.

\section{Method}

Participants. Undergraduate participants from a public Canadian University in Ontario were recruited throughout the 2016-2017 academic year for a larger study aimed at understanding academic performance, diversity, and well-being at the University. Only relevant scales to the theoretical position in this paper are reported. Instructors at the University who taught classes that involved group assignments were asked to post a message on their course's online learning platform, or to make announcements in their course to ask their students to participate in this study. Provided that nature of the study was to understand undergraduate 
student well-being, the sample size was not planned prior to data collection. Sample size was contingent upon trying to recruit as large of a sample as possible to represent the University's student body, and targeted students in classes with group assignments.

Procedure. Participants were asked to complete an online survey during a 2-week window at the start (Time 1; T1) and end of the academic term (Time 2; T2). Those who completed both $\mathrm{T} 1$ and $\mathrm{T} 2$ measures were entered into a raffle for a $\$ 50 \mathrm{CAD}$ cash prize. Of the 176 participants who completed the T1 measures at the start of the term, 94 also completed the T2 measures. Analyses involved changes in responses across the two time points, thus, the presented results are only from the participants who completed both $\mathrm{T} 1$ and $\mathrm{T} 2$ measures. The average amount of time between data collection at $\mathrm{T} 1$ and $\mathrm{T} 2$ was 59 days $(S D=9, M d n=56)$, with a range of 47 to 83 days. Participants began each survey at T1 and T2 by indicating which course they were completing this survey for, and then completed demographic questions assessing their gender, age, ethnic background, and social class.

Individualism measure. Participants completed the Singelis self-construal scale (Singelis, 1994) as an index of their individualistic values. The scale included five items assessing individualistic self-construal (e.g., "I enjoy being unique and different from others in many respects"). Items were presented in a randomized order and participants rated their agreement with each item on a scale from 1 (strongly disagree) to 7 (strongly agree). Mean responses were computed as an overall representation of individualism: Time 1: $M=4.91 S D=$ $.81, \alpha=.55$; Time $2: M=4.97, S D=.92, \alpha=.69$ ).

Ethnic diversity measure. Participants were asked to report their perception of the student's distribution of ethnic groups in their specific class. Using this information, a measure of EF used in Study 1 in the main text was computed as an index of ethnic diversity $(M=.67 ; S D$ 
$=.19$ ). As this study involved a Canadian sample, the ethnic groups were revised to reflect one used in the Canadian Census. Specifically, participants were provided with the following eight ethnic categories and reported their perception of the proportion of each ethnic group in their class (requiring a total of 100): White or European; ii. Black or African; iii. Hispanic, Latino, or Spanish origin; iv. Middle Eastern; v. East Asian; vi. South Asian; vii. Aboriginal; viii. Other.

\section{Results}

To test whether greater ethnic diversity at the start of the academic term predicted greater individualism at the end of the term, we first created a difference score between participants' endorsement of individualism at T2 and their endorsement of individualism at T1. T1 and T2 measures of individualism did not significantly differ from each other, with a mean difference of .04 between the two time points, $t(93)<1.00, p=.617$. EF at $\mathrm{T} 1$ showed a small-sized effect with a positive association to the $\mathrm{T} 2 \mathrm{vs}$. T1 difference score in individualism, $r=.19, p=.066$. This association suggests that greater ethnic diversity at $\mathrm{T} 1$ tended to predict an increased endorsement of individualism at T2, relative to participant's endorsement of individualism at T1.

To explore the direction of this association, we performed a cross-lagged panel analysis (Kearney, 2017), testing whether the perceptions of ethnic diversity in the classroom at the start of an academic term, predicted student's endorsement of individualistic values at the end of the academic term, controlling for each variable at the opposing time point. Perceptions of ethnic diversity at the start of the term was significantly related to perceptions of ethnic diversity at the end of the term, $r=.45, p<.001$. Agreement with individualism was also significantly related across the two time points, $r=.47, p<.001$. Given the equivalence of rank-order stability of each variable ( $r=.45$ vs. $r=.47$ ), and because more sophisticated analyses require more than two time points (Mund \& Nestler, 2019), we proceeded with a cross-lagged panel model 
analyses. Figure S2 depicts the model used for this analysis, which showed good model fit, $R M S E A=.036, P C L O S E=.649, C F I=.970$. Results from the cross-lagged panel analysis revealed that greater perceptions of ethnic diversity at the start of the academic term was significantly associated with greater individualistic values at the end of the term, $B=1.19, S E=$ $.54, p=.027$, whereas the reverse case of individualistic values at the start of the academic term, was not significantly associated with perceptions of ethnic diversity at the end of the term, $B=$ $.201, S E=.14, p=.152$. 


\section{Supplemental References}

Dhamo, E., \& Puka, L. (2010). Using the R-package to forecast time series: ARIMA models and Application. In International Conference of Economic \& Social Challenges and Problems 2010 Facing Impact of Global Crisis.

Jebb, A. T., \& Tay, L. (2017). Introduction to time series analysis for organizational research: Methods for longitudinal analyses. Organizational Research Methods, 20, 61-94.

Kearney, M. (2017). Cross-lagged panel analysis. The SAGE Encyclopedia of Communication Research Methods, 355, 165-174. https://doi.org/10.1002/jlac.19073550204

Kraus, M. W., Piff, P. K., \& Keltner, D. (2011). Social class as culture: The convergence of resources and rank in the social realm. Current Directions in Psychological Science, 20(4), 246-250. https://doi.org/10.1177/0963721411414654

Mund, M., \& Nestler, S. (2019). Beyond the cross-lagged panel model: Next-generation statistical tools for analyzing interdependencies across the life course. Advances in Life Course Research, 41, 100249. https://doi.org/10.1016/j.alcr.2018.10.002

Oyserman, D., Coon, H. M., \& Kemmelmeier, M. (2002). Rethinking individualism and collectivism: Evaluation of theoretical assumptions and meta-analyses. Psychological Bulletin, 181, 3-72.

Santos, H. C., Varnum, M. E. W., \& Grossmann, I. (2017). Global Increases in Individualism. Psychological Science, 28, 1228-1239. https://doi.org/10.1177/0956797617700622

Singelis, T. M. (1994). The measurement of independent and interdependent selfconstruals. Personality and Social Psychology Bulletin, 20, 580-591.

U.S. Census Bureau Data (2020). Historical Household Income Tables. Retrieved from https://www.census.gov/data/tables/time-series/demo/income-poverty/historical-incomehouseholds.html 
A

Original

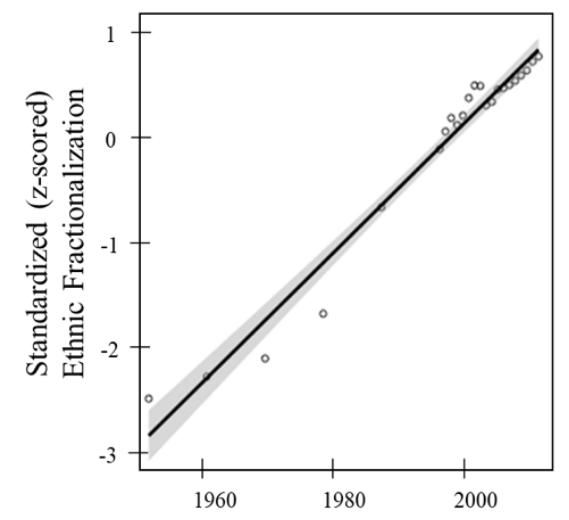

B

First-order detrended

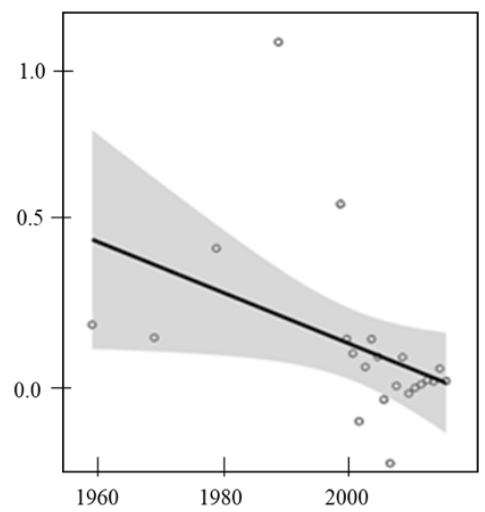

$\mathrm{C}$

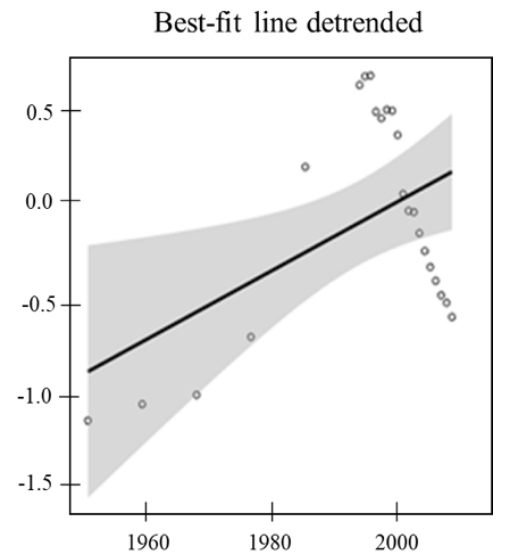

Figure S1. Ethnic diversity levels across the U.S. from the years 1950-2018 when not detrended (Panel A), detrended by removing first-order differences (Panel B), and detrended by removing the least squares fit from the original data (Panel C). 


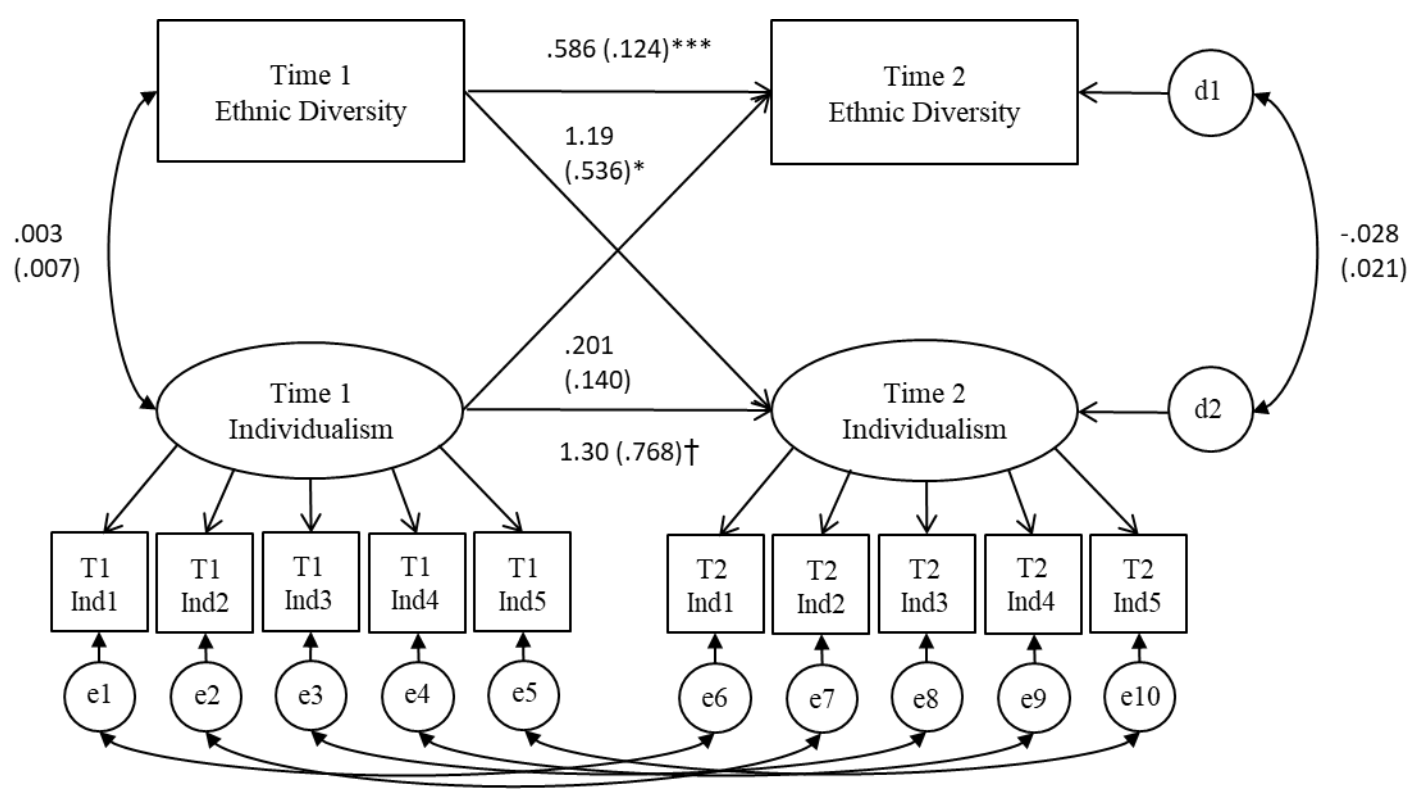

Figure S2. Path model of ethnic fractionalization and individualism across two different time points (see supplemental study). Individualism measures were classified as a latent variable from the Singelis' self-construal scale items at both time points. Numbers reflect the unstandardized estimates, with standard error in parentheses. Straight arrows represent regression paths and the curved arrows represent correlations. $\dagger p<.10, * p<.01, * * * p<.001$. 


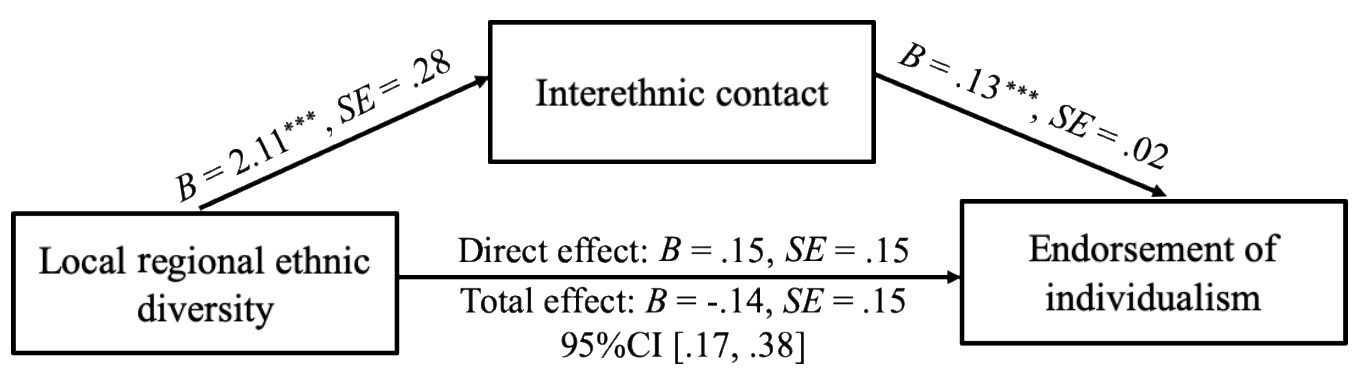

Figure S3. The indirect effect interethnic contact on participant's ethnic diversity in their local regions and their endorsement of individualism. 


\section{Table S1}

Study la correlation coefficients for ethnic diversity and household income with correlates of individualism

\begin{tabular}{|c|c|c|c|c|c|c|}
\hline \multirow[b]{2}{*}{$\begin{array}{l}\text { Correlates of } \\
\text { Individualism }\end{array}$} & \multicolumn{3}{|c|}{ Ethnic Diversity } & \multicolumn{3}{|c|}{ Household Income } \\
\hline & $\begin{array}{c}\text { Non- } \\
\text { detrended }\end{array}$ & $\begin{array}{l}\text { First- } \\
\text { order }\end{array}$ & $\begin{array}{l}\text { Best-fit } \\
\text { line }\end{array}$ & $\begin{array}{c}\text { Non- } \\
\text { detrended }\end{array}$ & $\begin{array}{l}\text { First- } \\
\text { order }\end{array}$ & $\begin{array}{c}\text { Best-fit } \\
\text { line }\end{array}$ \\
\hline Divorce/marriage ratio & $.78^{* * *}$ & $.30^{*}$ & $.88^{* * *}$ & $.74^{* * * *}$ & -.18 & .24 \\
\hline Adults living alone & $.67^{* * *}$ & .10 & $.46^{* *}$ & $.67^{* * *}$ & .12 & -.03 \\
\hline Smaller family size & $.83^{* * *}$ & .25 & $.81^{* * *}$ & $.78^{* * *}$ & $.32^{\dagger}$ & .18 \\
\hline Single-multi child & $.78^{* * *}$ & .16 & $.72^{* * *}$ & $.80^{* * *}$ & -.14 & .20 \\
\hline Averaged measure: & $.85^{* * *}$ & .23 & $.78^{* * *}$ & $.83^{* * *}$ & .01 & .24 \\
\hline
\end{tabular}

Note. Correlations represent the Kendall's $\tau$ coefficients for non-detrended data, and Pearson's $r$ for detrended data. Family size was reverse coded such that all positive number indicate a positive association with the correlates of individualism.

${ }^{\dagger} p<.10 ;{ }^{*} p<.05 ;{ }^{* *} p<.01 ;{ }^{* * *} p<.001$ 


\section{Table S2}

Study $1 \mathrm{~b}$ estimates of fixed effects from multi-level modeling ethnic diversity and correlates of individualism (top rows), as well as ethnic diversity and household income simultaneously predicting correlates of individualism (bottom rows)

\begin{tabular}{lccc} 
& & \multicolumn{2}{c}{ Ethnic Diversity } \\
\hline Correlates of Individualism & Intercept & \multicolumn{2}{c}{$B(\mathrm{SE})$} \\
\hline Divorce/marriage ratio & -.13 & \multicolumn{2}{c}{$.18(.03)^{* * *}$} \\
Adults living alone & -.11 & \multicolumn{2}{c}{$.23(.03)^{* * *}$} \\
Smaller family size & -.04 & \multicolumn{2}{c}{$.37(.04)^{* * *}$} \\
Single/multi-child families & -.03 & \multicolumn{2}{c}{$.33(.04)^{* * *}$} \\
\multicolumn{1}{c}{ Averaged measure: } & $-.10^{\ddagger}$ & \multicolumn{2}{c}{$.24(.03)^{* * *}$} \\
\hline Correlates of Individualism & & Ethnic & Household \\
\hline Divorce/marriage ratio & Intercept & Diversity & Income \\
Adults living alone & -.11 & $.13(.03)^{* * *}$ & $.27(.04)^{* * *}$ \\
Smaller family size & $-.10^{*}$ & $.14(.03)^{* * *}$ & $.38(.04)^{* * *}$ \\
Single/multi-child families & -.03 & $.14(.05)^{* *}$ & $.39(.05)^{* * *}$ \\
\multicolumn{1}{c}{ Averaged measure: } & -.02 & $.18(.05)^{* * *}$ & $.26(.05)^{* * *}$ \\
\hline & -.08 & $.16(.03)^{* * *}$ & $.31(.03)^{* * *}$ \\
\hline
\end{tabular}

${ }^{7} p<.10 ;{ }^{* *} p<.01 ;{ }^{* * *} p<.001$ 